\title{
Controlled substances and innovation of biomedicine: a preclinical perspective
}

\author{
Adam Michael Stewart and Allan V. Kalueff
}

In their recent article (Effects of Schedule I drug laws on neuroscience research and treatment innovation. Nature Rev. Neurosci. 14, 577-585 (2013)) $)^{1}$, Nutt and colleagues show how the over-regulation of controlled substances lacks practical or scientific reasoning, hinders treatment innovation and shifts focus from curbing drug abuse to fighting substances that are less harmful and less addictive. Continuing this important and timely discussion, we evaluate the impact of drug laws on experimental (basic) research.

Animal models are indispensable for neuropsychiatric research ${ }^{2}$. Addressing 'grand' challenges in mental health ${ }^{3}$ (Supplementary information S1 (box)), basic studies of hallucinogens may alleviate human suffering ${ }^{4-6}$ by uncovering evolutionarily conserved mechanisms of drug-evoked brain disorders. However, over-reaching controlled-substance laws markedly limit this field. For example, classifying drugs under Schedule I creates negative bias, so that animal studies are often deemed as 'less clinically relevant' if they focus on psychedelic rather than on more traditional, well-studied substances. Applying for special licences is also particularly arduous for basic scientists in the field ${ }^{1}$. Although many researchers may wish to study hallucinogens ${ }^{7,8}$, time-consuming US Food and Drug Administration (FDA) approval is required for the federal Drug Enforcement Administration (DEA) licence, followed by licensing by the States' Boards of Pharmacy. Any new drug must be 'added' to the licence, which also takes time and leads to 'interesting' complications. For example, after our recent order of tetrahydrocannabinol resulted in approval for drug-containing cigarettes, it took an additional 4 weeks (with new paperwork and calls to various offices to explain that animals do not smoke) to obtain the ethanol-based drug solution.

The lack of reasonable and affordable access to psychotropic drugs is another obstacle for basic researchers. For example, 3,4-methylenedioxy- $N$-methylamphetamine from Sigma-Aldrich costs over US $\$ 400$ for $50 \mathrm{mg}$, or over $\$ 7000$ for a single-dose zebrafish experiment ${ }^{9}$. Another problem is the availability of certain controlled drugs. Consider ibogaine, an extremely potent, unique anti-addictive agent that combines pharmacological mechanisms of all known hallucinogens ${ }^{10}$. Currently, ibogaine is not produced by any US commercial vendor except for the US National Institute on Drug Abuse (NIDA) Drug Supply Program (Supplementary information S2 (box)). Without a Schedule I licence and access to this US National Institutes of Health (NIH) resource, our animal research ${ }^{10}$ would have been impossible - the misfortune of many laboratories worldwide where ibogaine is not offered by biomedical suppliers. Clearly, critical new knowledge can go undiscovered because of such limitations on translational preclinical drug research.

In summary, the growing interest in studying psychotropic drugs meets dated over-regulation that hinders translational biomedical research. As basic scientists, we are joining clinicians ${ }^{1}$ to increase public awareness of the current situation and convince legislators to remove unnecessary obstacles in this field. Potential practical solutions may include removing hallucinogens from Schedule I and/or placing all controlled drugs that are specifically used in basic research into a separate category (for example, in a 'Schedule VI' created especially for research-grade drugs). Finally, governments and funding bodies must encourage further basic studies of both the negative and positive effects of psychotropic controlled substances. Studying anti-addictive mechanisms of the hallucinogenic drug ibogaine is just one of many examples of how preclinical studies may offer important insights in this field. Despite the efforts of a few dedicated scientists ${ }^{1}$, new treatments for psychiatric disorders will continue to be greatly hindered unless the current stranglehold on generating clinical and basic knowledge is alleviated.

Adam Michael Stewart and Allan V. Kalueff are at the ZENEREI Institute, Slidell, Louisiana 70458, USA.

Adam Michael Stewart is also at the Department of Neuroscience, University of Pittsburgh, Pittsburgh, Pennsylvania 15260, USA.

Correspondence to A.V.K. e-mail: avkalueff@gmail.com

doi:10.1038/nrn3530-c1 Published online 23 October 2013

1. Nutt, D. J., King, L. A. \& Nichols, D. E. Effects of Schedule I drug laws on neuroscience research and treatment innovation. Nature Rev. Neurosci. 14 577-585 (2013).

2. Nestler, E. J. \& Hyman, S. E. Animal models of neuropsychiatric disorders. Nature Neurosci. 13 1161-1169 (2010).

3. Collins, P. Y. et al. Grand challenges in global mental health. Nature 475, 27-30 (2011).

4. Geyer, M. A. Why study hallucinogenic drugs in animals? Heffer Rev. Psychedel. Res. 1, 33-38 (1998)

5. Moghaddam, B. \& Krystal, J. H. Capturing the angel in "angel dust": twenty years of translational neuroscience studies of NMDA receptor antagonists in animals and humans. Schizophrenia Bull. 38, 942-949 (2012).

6. Krystal, J. H., Anand, A. \& Moghaddam, B. Effects of NMDA receptor antagonists: implications for the pathophysiology of schizophrenia. Arch. Gen. Psychiatry 59, 663-664 (2002).

7. Geyer, M. A. \& Vollenweider, F. X. Serotonin research: contributions to understanding psychoses. Trends Pharmacol. Sci. 29, 445-453 (2008).

8. Hanks, J. B. \& Gonzalez-Maeso, J. Animal models of serotonergic psychedelics. ACS Chem. Neurosci. 4, 33-42 (2013)

9. Stewart, A. et al. Behavioral effects of MDMA ("Ecstasy") on adult zebrafish. Behav. Pharmacol. 22, 275-280 (2011)

10. Cachat, J. et al. Unique and potent effects of acute ibogaine on zebrafish: the developing utility of novel aquatic models for hallucinogenic drug research. Behav. Brain Res. 236, 258-269 (2013).

Acknowledgements

The authors acknowledge the important role of US National Institute on Drug Abuse Drug Supply Program of the US National Institutes of Health in providing the costly controlled substances free of charge, including supplying ibogaine (which we were unable to obtain from any other sources).

Competing interests statement

The authors declare no competing interests.

SUPPLEMENTARY INFORMATION

See online article: $\underline{\mathrm{S}}$ (box) | $\underline{\mathrm{S} 2}$ (box)

ALL LINKS ARE ACTIVE IN THE ONLINE PDF 